\title{
The 'tail' of a missing intrauterine contraceptive device
}

Hanan Ismail, MB ChB, DFFP, Career Grade Trainee in Family Planning and Reproductive Health, Graingerville Clinic, Newcastle General Hospital, Newcastle upon Tyne, UK; Diana Mansour, FRCOG, MFFP, Consultant in Community Gynaecology and Reproductive Health Care, Graingerville Clinic, Newcastle General Hospital, Newcastle upon Tyne, UK

Correspondence: Hanan Ismail, Graingerville Clinic, Newcastle General Hospital, Westgate Road, Newcastle upon Tyne NE4 6BE, UK. Tel: +44 (0) 191219 5239. Fax: +44 (0) 1912195232

(Accepted $21^{\text {st }}$ January 2002)

The Journal of Family Planning and Reproductive Health Care 2002: 28(2): 96-97

\section{Abstract}

This case presents a cautionary tale demonstrating the importance of skilled ultrasonography for the correct location of an intrauterine device (IUD) lying within the uterine cavity where the threads are 'lost'. Accurate ultrasound examination can ensure the avoidance of unnecessary $x$-rays or surgery. The case also acts as a reminder that a missing thread of an IUD does not imply that the device is misplaced.

\section{Case report}

A woman attended the contraception and sexual health clinic requesting to use a copper intrauterine device (IUD) as her method of contraception. The woman was fit, well and taking no medication. She had one child, a boy, aged 2 years. She had previously taken a combined oral contraceptive (COC) pill but had experienced recurrent migraines. The progestogen-only pill (POP) had made her feel depressed. Therefore, an IUD was felt to be a suitable choice. Following counselling and endocervical screening for sexually transmitted infections (STIs), a Multiload Cu375 IUD was fitted on Day 7 of her menstrual cycle. The IUD fitting was straightforward and unremarkable.

At a routine check-up 6 weeks after insertion, the IUD threads were visible. Two months after insertion, the patient presented to the clinic complaining of a vaginal discharge. On examination, no pelvic tenderness or other abnormalities were found. The IUD threads were again visible and endocervical swabs were taken. The swabs proved negative and her symptoms resolved without treatment.

Six months after insertion the patient presented to the clinic and requested removal of the IUD as she wished to have another baby. The patient was well and had no complaints. Her last menstrual period had started 3 days prior to the clinic appointment. At pelvic examination the IUD threads were not visible. An attempt at removal was undertaken using long-handled Spencer Wells forceps and a thread retriever. These manoeuvres failed to locate the IUD threads, therefore an ultrasound scan of the pelvis was requested (Figure 1 ).

The radiographer stated that she was unable to demonstrate the IUD in the uterine cavity, therefore an abdominal x-ray was performed. The IUD was identified in the midline of the pelvis (Figure 2). Arrangements were therefore made for a consultation with the community gynaecologist to discuss further management. The patient
Figure 1 First pelvic ultrasound [transvaginal (TV) scan]: the IUD was not identified within the uterine cavity on ultrasound

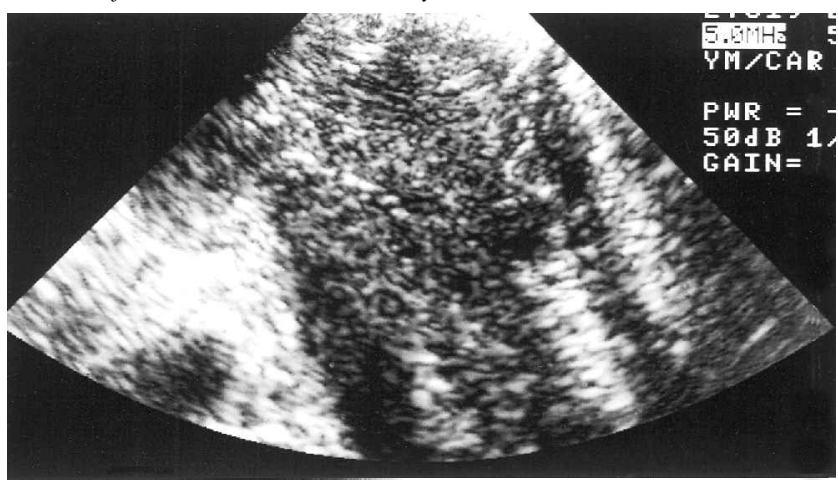

Figure 2 Pelvic $x$-ray: the IUD is identified in the midline in the pelvis but it is not possible to be certain of its exact location in relation to the uterus

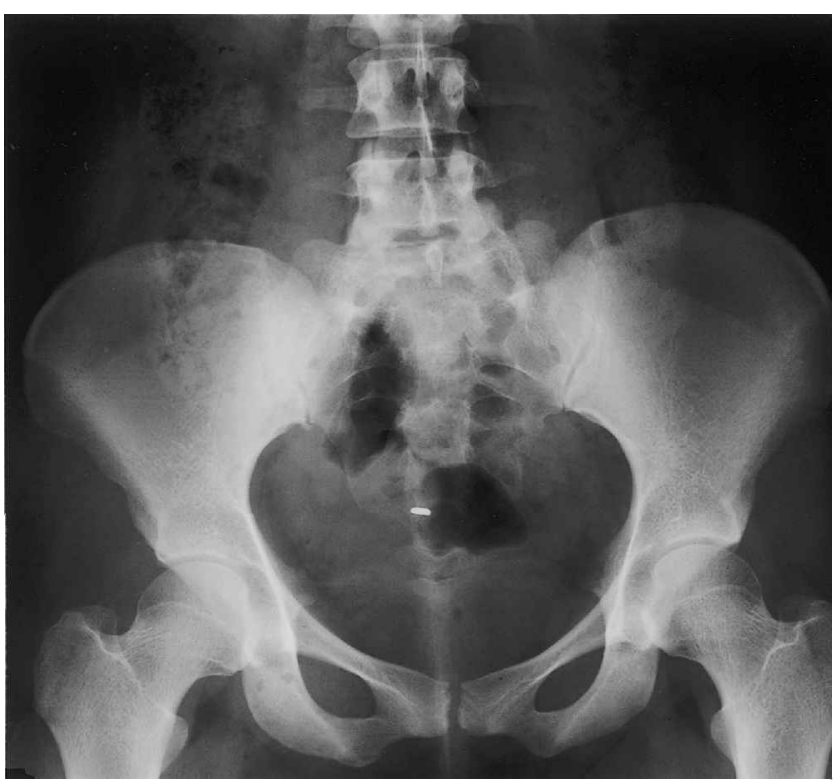

was aware that a laparoscopy was indicated and there was a possibility of this leading to a laparotomy.

The pelvic ultrasound scan and the x-ray film were reviewed by the community gynaecologist who advised that the ultrasound scan should be repeated. The IUD appeared on the X-ray film to be centrally placed in the pelvis but the initial pelvic ultrasound was of such poor quality that one was unable to draw any firm conclusions. A repeat pelvic ultrasound scan performed using a portable machine in the 
Figure 3 Second pelvic ultrasound [transabdominal (TA) scan]: the IUD can be seen within the uterine cavity which appears to be correctly positioned

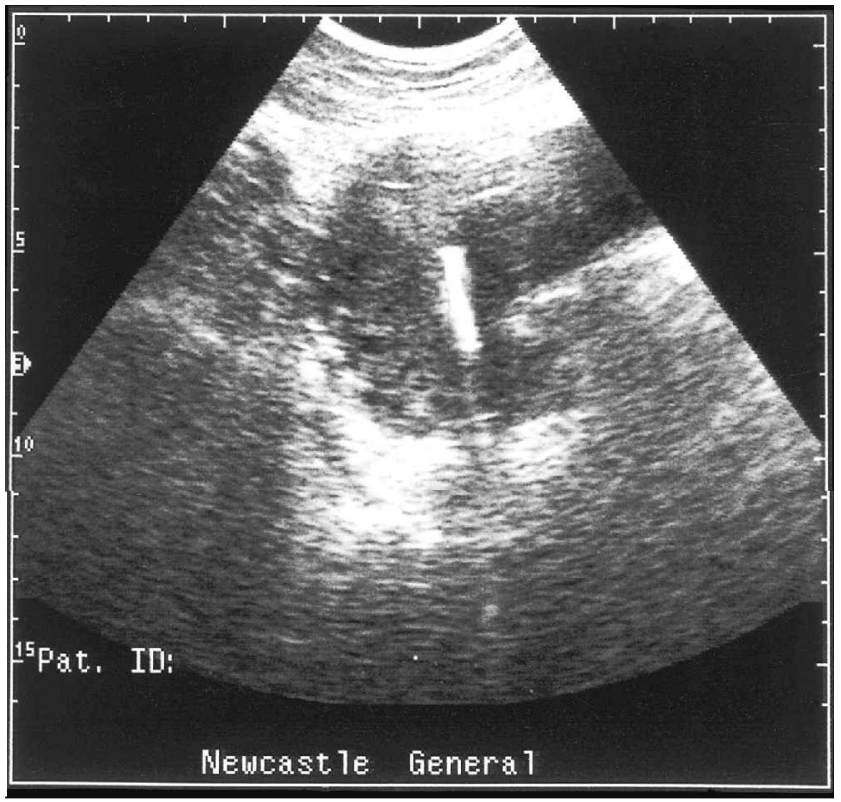

Figure 4 Alligator forceps (Rocket, Watford, UK)

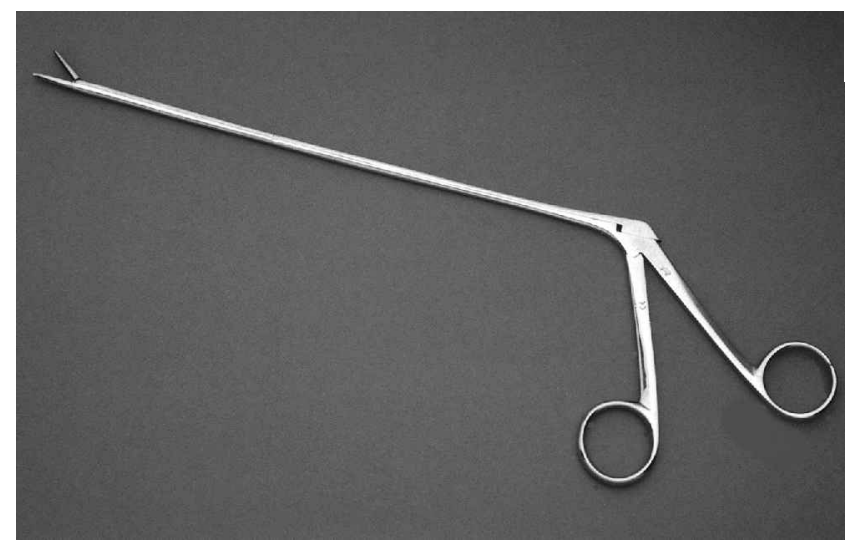

central clinic clearly identified the IUD lying within the uterine cavity (Figure 3). The IUD was removed easily with Alligator forceps (Figure 4). These forceps have serrated jaws and open in one direction. The forceps were introduced through the cervix. The jaws opened to grasp the IUD, which was then removed.

\section{Discussion}

This case report describes a patient in whom the marker thread of her IUD was not visible on speculum examination at follow-up. She had been fitted with a Multiload Cu375 IUD and attended 6 months subsequently requesting removal in order to embark on a pregnancy.

The Multiload Cu375 is an IUD with flexible side arms and made from a mixture of high-density polyethylene and barium sulphate. A copper wire is wound around its vertical stem. The addition of the barium sulphate allows the plastic part (side arms) of this device to be located by x-ray. ${ }^{1}$

In cases where the marker thread of an IUD disappears there are three possibilities. First, that there may have been unrecognised spontaneous expulsion. Second, that the thread of the IUD may be coiled up and lying within the endocervix. Third, that the IUD may have perforated the uterine wall and transmigrated into the peritoneal cavity.

In the management of such a case general principles dictate that the endocervix should be explored with Spencer Wells forceps to determine whether the thread of the IUD was coiled up at that site. In the present case this manoeuvre was undertaken without success.

Although some authorities ${ }^{2}$ recommend uterine sounding and/or gentle exploration of the uterine cavity with a thread retriever this was not carried out in this case, because it was considered too painful, and because facilities for ultrasound examination were easily accessible.

However, the first ultrasound examination failed to identify the IUD thus necessitating an abdominal x-ray which was indeed in accord with general principles in the management of lost IUD threads.

The positive finding of the IUD on abdominal x-ray and the negative report of the first ultrasound examination suggested perforation and transmigration. Accordingly laparoscopy and possible laparotomy were contemplated.

This intervention was averted by reviewing the woman's clinical history since the time of IUD insertion. There was no clinical suspicion of perforation. Accordingly a second ultrasound examination was performed, but on this occasion it was carried out by a skilled and experienced operator.

Uterine perforation has been reported to depend on the type of IUD, the timing of insertion relative to the time from termination of pregnancy (post-abortion or postpartum), the insertion technique and the skill of the individual inserting the device. ${ }^{3}$ The device was fitted in a family planning clinic by a skilled individual. It was not fitted post-abortion or postpartum. One has to accept, however, that even in skilled hands perforation can occur.

The key issue in this patient's case was the avoidance of unnecessary intervention primarily due the accuracy of the second ultrasound examination. It is clear from the outcome in this patient's case that the first ultrasound examination was carried out by a less skilled operator. We recommend that only trained staff should undertaken ultrasound examinations in clinical settings.

There are only a limited number of courses available to provide gynaecology ultrasound training. It would be advantageous if these courses could be more widely publicised and perhaps for the future, receive recognition from the Faculty of Family Planning and Reproductive Health Care of the Royal College of Obstetricians and Gynaecologists.

Had the first ultrasound examination been more accurate it is possible that the abdominal $\mathrm{x}$-ray could have been avoided. Had the second ultrasound not been performed this patient would have been subjected to unnecessary laparoscopy and possible laparotomy.

Statements on funding and competing interests

Funding. None declared.

Competing interests. None declared.

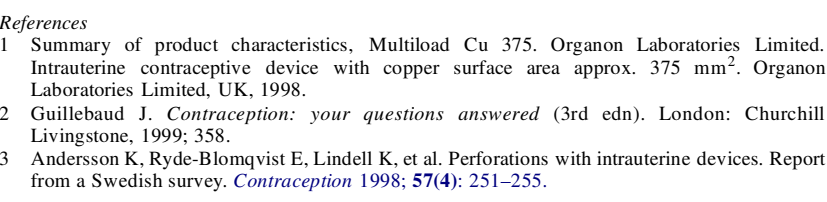

